\title{
Anterior thalamic lesions alter both hippocampal- dependent behavior and hippocampal acetylcholine release in the rat
}

\author{
Lisa M. Savage, ${ }^{1}$ Joseph M. Hall, and Ryan P. Vetreno \\ Behavioral Neuroscience Program, Department of Psychology, State University of New York at Binghamton, Binghamton, \\ New York 13902, USA
}

\begin{abstract}
The anterior thalamic nuclei (ATN) are important for learning and memory as damage to this region produces a persistent amnestic syndrome. Dense connections between the ATN and the hippocampus exist, and importantly, damage to the ATN can impair hippocampal functioning. Acetylcholine $(\mathrm{ACh})$ is a key neurotransmitter in the hippocampus, and in vivo measures of $\mathrm{ACh}$ are correlated to learning and memory performance. In the present study, complete lesions of the ATN impaired performance on two measures of hippocampal-dependent learning and memory (spontaneous alternation and delayed alternation) and severely disrupted behaviorally evoked ACh efflux within the hippocampus of adult male rats. In contrast, incomplete ATN lesions did not impair spontaneous alternation performance but did impair delayed alternation performance while blunting hippocampal ACh efflux. Interestingly, ATN lesions of any size did not affect basal concentrations of ACh in the hippocampus. These results demonstrate that the ATN have the capacity to modulate behaviorally relevant neuronal transmission within the hippocampus.
\end{abstract}

Diencephalic amnesia, produced by thiamine deficiency or thalamic infarction, is consistently associated with neuropathology in the anterior and midline thalamic nuclei, mammillary bodies, and/or the mammillothalamic tract (Mair 1994; Harding et al. 2000; Van der Werf et al. 2003; Gold and Squire 2006; Aggleton et al. 2011; Carlesimo et al. 2011). Although the severity of cognitive dysfunction is likely related to the aggregate of pathology across multiple diencephalic nuclei, the anterior thalamic nuclei (ATN) are emerging as a critical diencephalic candidate as they influence both spatial and recognition memory (Aggleton et al. 2011). Indeed, lesion of the rodent ATN degrades performance on hippocampal-dependent spatial memory tests (Sutherland and Rodriguez 1989; Aggleton and Sahgal 1993; Byatt and Dalrymple-Alford 1996; Sziklas and Petrides 1999). Furthermore, contralateral disconnection studies strongly support the hypothesis that the hippocampus and the ATN are reciprocally connected and are dependent on each other to produce successful spatial learning and memory (Warburton et al. 2000, 2001). However, another way to assess the interactions between brain regions is to assess how damage in one structure influences neural processing in the other region.

Traditionally, the focus on the interaction between the ATN and the hippocampus has been hippocampal-centric, i.e., that hippocampus modulates ATN function. However, there is an emerging view that postulates that the ATN can impact hippocampal and limbic-cortical functioning (see Aggleton et al. 2010; Vann 2010). Neuroanatomical connectivity produces the architecture for the ATN and the hippocampus to directly and indirectly modulate the neural function of one another. The hippocampus projects to the ATN both directly via the fornix and indirectly through the mammillary bodies and mammillothalamic tract (Swanson and Cowan 1977). In a similar fashion, the ATN project directly to the hippocampus through the cingulum

\footnotetext{
${ }^{1}$ Corresponding author.
}

E-mail Isavage@binghamton.edu.

Article is online at http://www.learnmem.org/cgi/doi/10.1101/lm.023887.111. bundle (Shibata 1993) and indirectly via the retrosplenial cortex and mammillary bodies (Mufson and Pandya 1984; Van Groen and Wyss 1995). Thus, these structures display the capacity to regulate the neural functions of each other in a bidirectional manner.

Consistent with the reciprocal connectivity hypothesis, several studies have shown that both bilateral and unilateral lesions of the ATN reduce behaviorally activated expression of the immediate early gene c-Fos in many hippocampal subfields (Jenkins et al. 2002a,b), with the dorsal (septal) hippocampus showing the greatest reduction (Vann and Albasser 2009). Electrophysiological evidence has also revealed that damage to the ATN can disrupt hippocampal neural activity. For instance, lesions to the anterior dorsal thalamic nuclei (AD) result in a loss of headdirection cells in the postsubiculum, but the opposite pattern of results was not observed (Goodridge and Taube 1997). In addition, the anterior ventral thalamic nuclei (AV) have theta oscillations that correlate with hippocampal theta rhythm (Vertes et al. 2004; Tsanov et al. 2011). These results have led the authors to suggest that the AV nuclei are part of a descending theta system driven from the medial septum that facilitates hippocampaldependent memory processing. At this point, it is unknown whether a lesion of the ATN disrupts hippocampal theta oscillations.

Using the pyrithiamine-induced thiamine deficiency model (PTD) model of diencephalic amnesia, we have demonstrated reduced hippocampal acetylcholine $(\mathrm{ACh})$ efflux when rats are tested on hippocampal-dependent tasks (Savage et al. 2003; Roland and Savage 2007; Vetreno et al. 2008). However, the PTD model recapitulates the diverse neuropathology common to Korsakoff Syndrome, which includes several anterior, medial, and lateral thalamic nuclei, as well as the mammillary bodies and interconnecting fiber tracts. Moreover, in the PTD model, there is a loss of cholinergic cells and fibers that innervate the hippocampus as well as reduced hippocampal neurogenesis (Roland and Savage 2009; Anzalone et al. 2010; Vetreno et al. 2011). Thus, both the loss of neurons in the diencephalic nuclei and specific loss of cholinergic neurons in the medial septal/diagonal 
band (MS/DB) could contribute to the behavioral impairments and blunted behaviorally evoked hippocampal ACh efflux in the PTD model. However, at this point we do not know the individual contributions of these neuropathological features to the hippocampal cholinergic dysfunction observed in the PTD model of Korsakoff Syndrome. The lesion approach in the current experiment should provide some resolution on this issue.

Other studies using the discrete lesion approach have demonstrated that damage to the ATN has the capacity to disrupt hippocampal electrophysiological resting states (Gabriel et al. 1995) and behaviorally activated states (Jenkins et al. 2002a). The in vivo microdialysis procedure used in the current experiment allows for the assessment of neurochemical changes across different behavioral states. Acetylcholine is a good measure of neural activation for two reasons: First, in behaving animals, high ACh levels have been hypothesized to repress activation of intrahippocampal pathways but facilitate hippocampal-cortical communication to promote the encoding of spatial or contextual information (Hasselmo 2006). Thus, low hippocampal ACh levels, prior to or during learning, tend to be associated with impaired learning of spatial relationships (McIntyre et al. 2003; Savage et al. 2003). It has been stated that the level of ACh in a given memory structure reflects the activation of that structure during memory processing (Gold 2004). Second, ATN damage does not directly alter cholinergic innervation of the hippocampus. The ATN, primarily the AV nuclei, receive cholinergic input from the brainstem, whereas the hippocampus receives cholinergic innervation from the MS/ DB (Woolf 1991). Thus, disruption of this system would reveal a broad rather than specific impact on hippocampal functioning.

The current experiment assessed the effects of total and partial lesion of the ATN on the hippocampal-dependent behaviors of spontaneous alternation and delayed alternation. Furthermore, we measured ACh efflux in awake rats prior to, during, and after delayed alternation testing. We expected that hippocampal-dependent behaviors would be compromised by ATN lesions, and furthermore, such lesions would have the capacity to disrupt hippocampal neurotransmission as reflected by blunted ACh efflux.

\section{Statistical analyses}

The mean percent alternation and total number of arm entries during spontaneous alternation testing were analyzed with a one-factor (Group=sham, partial ATN lesion, complete ATN lesion) ANOVA. For the delayed alternation data, a mixed model design of a one-between subject factor (Group) and one-within subject factor of Session $(1,2)$ repeated measures ANOVA was used. Acetylcholine efflux data were analyzed with a mixed model ANOVA design that had one-between subjects factor (Group) and three-within subjects factors (Session [1, 2], Phase [baseline, maze, after], sample Time [block 1-3]).

\section{Results}

\section{Lesion and cannula placement}

Lesion verification was determined across several coronal sections that underwent NeuN immunohistochemistry, and subjects not meeting criteria for complete or partial AT lesions were excluded from analyses. Figure 1 depicts the largest and smallest acceptable ATN lesions for the complete ATN lesion group (A-C) and the partial ATN lesion group (D-F). Complete lesions of the ATN were defined as at least $65 \%$ bilateral destruction of all three principle anterior thalamic nuclei: anterodorsal (AD), anteroventral mediodoral (AVDM), and anteroventral-ventrolateral (AVVL). The complete ATN lesion ran the rostal-caudal extent of the regions (from $\sim 7.7 \mathrm{~mm}$ to $\sim 7.2 \mathrm{~mm}$, interaural according to the atlas of Paxinos
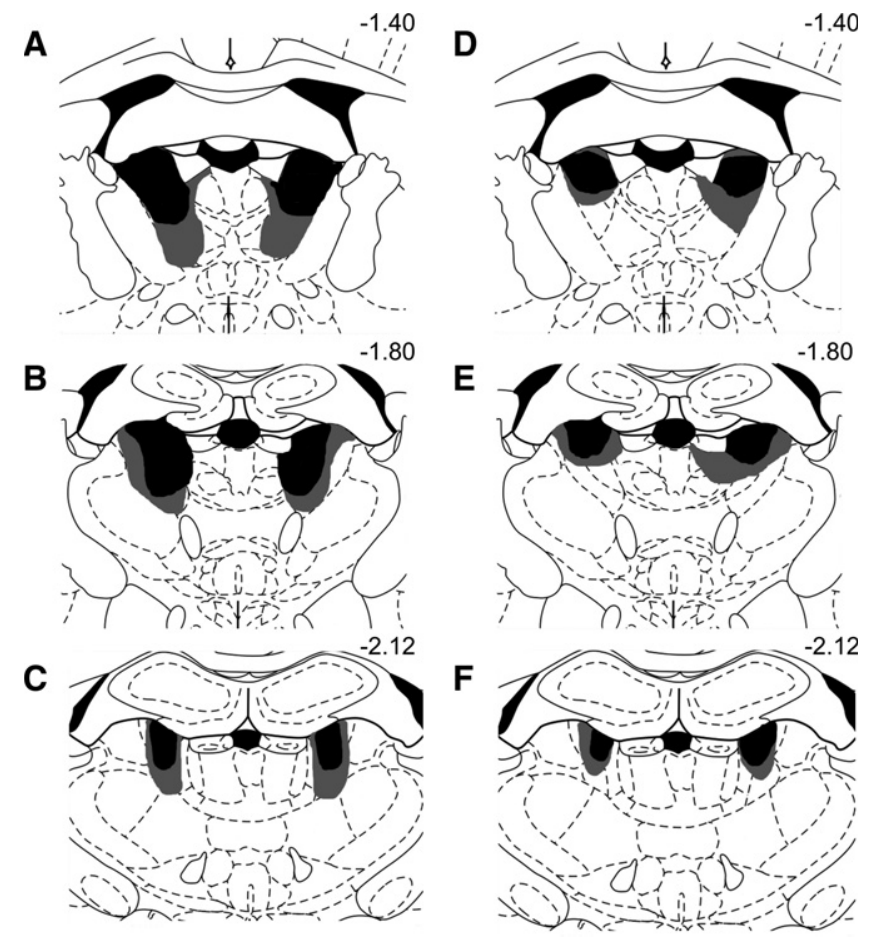

Figure 1. Series of coronal illustrations depicting anterior thalamic cell loss in the smallest (black) and largest (gray) lesion. Lesions are shown in both the complete AT lesion ( $A-C$ on the left) and partial AT lesion $(D-F$ on the right). The series distance from bregma is reported numerically in millimeters. Note that damage is largely restricted to AT nuclei. Also note that in the ATN partial lesion condition, damage to the AVVL was unilateral in $\sim 40 \%$ of the cases.

and Watson [2004]), but there was no evidence of midline thalamic damage. Thus, the medial dorsal and more caudal midline intralaminar nuclei were spared. A partial ATN lesion was defined as damage that did not encompass all three principle anterior thalamic nuclei bilaterally. For the majority of rats categorized as ATN partial (85\%), there was $\sim 60 \%$ damage to the AD, $50 \%$ damage to ADV, but AVVL was not greatly affected ( $\sim 40 \%$ bilateral, or only unilateral). Again, the midline thalamic nuclei were intact. Two subjects included in analyses contained damage to the parataenial nuclei; however, involvement was only minimal $(<20 \%)$. Representative brain sections that were stained with the immunomarker for NeuN from the sham (A), partial ATN (B), and complete ATN (C) lesion groups are shown in Figure 2. In addition, separate brain sections were stained with Pyronin Y to ensure correct hippocampal cannula placement (see Fig. 2D). The location of the microdialysis cannula was in the ventral hippocampus among the CA sectors (CA1, CA2, CA3). At the completion of the experiment, the final subject counts per group were: sham $=10 ;$ ATN partial $=7 ;$ ATN complete $=9$. Four rats had damage outside of the ATN or a misplaced hippocampal cannula and were not included in any analyses.

\section{Spontaneous alternation}

As depicted in Figure 3A, no significant differences in activity rates (number of arms entered) during maze training were observed as a function of Group $\left(F_{(2,23)}<1.59, P>0.20\right)$. However, Figure 3B reveals that there were significant differences in spontaneous alternation rates as a function of Group $\left(F_{(2,23)}=8.49, P<0.001\right)$. Post-hoc analyses (Fisher's LSD) demonstrated that there was a 
A

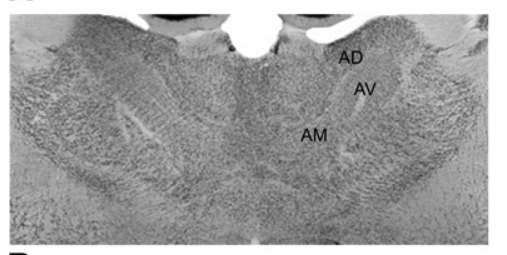

B

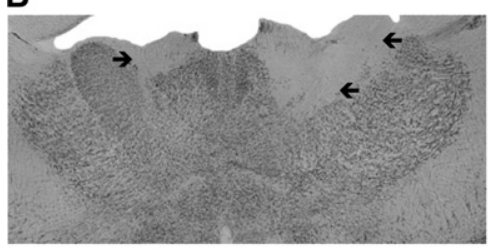

C

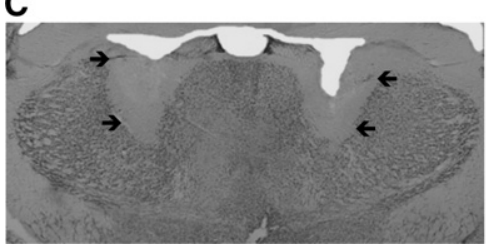

Figure 2. Photomicrograph of NeuN stained coronal sections showing the anterior thalamic nuclei from representative brain images from rats in the sham $(A)$, partial anterior thalamus lesion $(B)$, and complete anterior thalamus lesion $(C)$ groups. The partial and complete lesions differ in lesion size and location. A complete lesion had to have damage to both $A D$ and $A V$ nuclei. Note that the medial and midline regions are spared in both lesion groups. Arrows on images $B$ and $C$ outline lesion extent. (D) Hippocampal probe placement. Arrows depict end of cannula and microdialysis probe tract. (AD) Anterodorsal thalamic nucleus, (AV) anteroventral thalamic nucleus, (AM) anteromedial thalamic nucleus, (DG) dentate gyrus, (CA) cornu ammonis area.

substantial deficit in spontaneous alternation scores in rats with complete ATN lesions, relative to the sham group $(P<0.001)$ and the ATN partial lesion group $(P<0.05)$. However, the partial ATN group did not show a significant impairment, compared to sham rats $(P>0.30)$.

\section{Delayed alternation}

Behavior did not change across the two sessions of delayed alternation testing (both $F^{\prime}$ 's $<1.0$ ); thus, data were collapsed across sessions for further analyses. Figure $4 \mathrm{~A}$ illustrates that there were no significant Group differences in the number of arm entries during delayed alternation testing $\left(F_{(2,23)}<1.0\right)$. However, as shown in Figure $4 \mathrm{~B}$, there were significant differences as a function of Group for delayed alternation accuracy scores $\left(F_{(2,49)}=6.36, P<\right.$ $0.01)$. Fisher's LSD post-hoc analyses revealed a robust impairment of delayed alternation behavior in the complete ATN group, compared to the sham group $(P<0.01)$. Interestingly, the partial ATN group also displayed impaired alternation behavior relative to the sham group $(P<0.01)$. Furthermore, the partial ATN group was not significantly different from the complete ATN group $(P>0.50)$.

\section{Hippocampal ACh efflux}

As shown in Figure 5A, basal ACh levels in the hippocampus did not significantly differ as a function of Group $\left(F_{(2,49)}<1.0\right)$. Thus, damage to the ATN did not reduce overall cholinergic tone within the hippocampus. However, there were significant Group differences in the behaviorally evoked changes in ACh efflux $\left(F_{(2,23)}=7.95, P<0.01\right)$. Given that there was no main effect of Session $\left(F_{(1,23)}=1.64, P>0.20\right)$, the data are shown collapsed across the two delayed alternation sessions in Figure $5 \mathrm{~B}$. These Group differences were only detected as a function of Phase (Group $\times$ Phase interaction: $F_{(4,46)}=7.05, P<0.001$ ).

The microdialysis data revealed a greater rise in ACh efflux during maze testing in the sham group relative to both ATN lesion groups (both $P^{\prime}$ s $<0.025$ ). No other interactions were significant (all $P^{\prime}$ s $>0.05$ ). In the sham group during maze testing, there was an approximate $80 \%$ increase in ACh levels over the baseline level $\left(F_{(2,18)}=30.58, P<0.0001\right)$. However, the behaviorally driven ACh efflux was dramatically reduced in the ATN lesion groups: The partial ATN group had a rise that was less than $50 \%$ of the sham group $\left(F_{(1,15)}=8.01, P<0.05\right)$, but this behaviorally driven rise $(32 \%)$ was still significantly above the basal level $\left(F_{(2,12)}=17.56, P<0.001\right)$. The severely blunted behavioralinduced rise (17\%) in hippocampal ACh efflux in the complete ATN group was significantly above basal levels $\left(F_{(2,16)}=3.82\right.$, $P<0.05)$, but the rise was dramatically reduced relative to the sham group (only $\sim 20 \%$ of that observed the sham group; $\left.F_{(1,17)}=12.18, P<0.01\right)$. Thus, we see behaviorally driven activation, but not basal concentrations, of the hippocampal ACh system are dysfunctional after partial or complete lesions of the ATN.

\section{Correlation between behavior, lesion extent, and hippocampal ACh efflux}

Since there was no main effect of session on either behavior or ACh release, the data were pooled across the two sessions for the correlation analysis. The correlations between behavioral accuracy, lesion extent, and/or ACh release during maze testing for all groups of rats, or for just the ATN lesion groups, were conducted using simple regression models. As shown in Figure 6A, it was found that the average percent correct for delayed alternation was positively correlated $(r=0.44, P<0.03)$ with the ACh efflux during maze testing when all groups were included. However, if only the two groups with ATN lesions were used, there

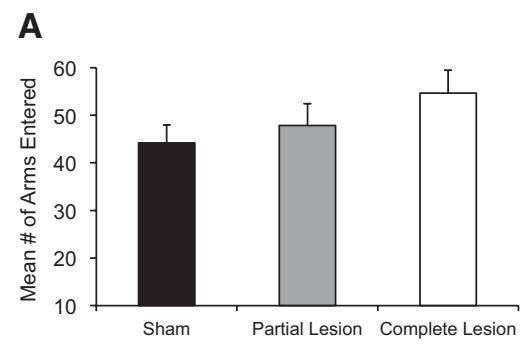

B

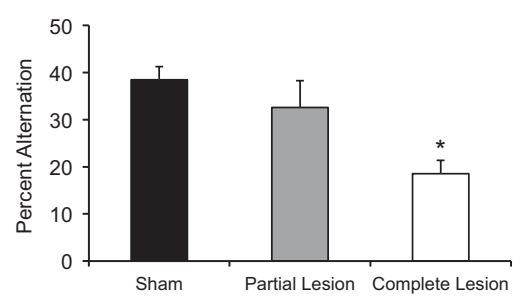

Figure 3. Spontaneous alternation behavioral results. (A) Activity data. The sham and lesion groups did not display any differences in activity as measures by the mean number of arms entered during the 18-min testing session. $(B)$ Percent spontaneous alternation. In contrast, rats with complete lesions of the anterior thalamus had significantly lower alternation scores relative to sham-treated rats or rats with partial lesions to the anterior thalamus, which were not different from each other. $\left(^{*}\right)$ Significant difference from sham group. 
A

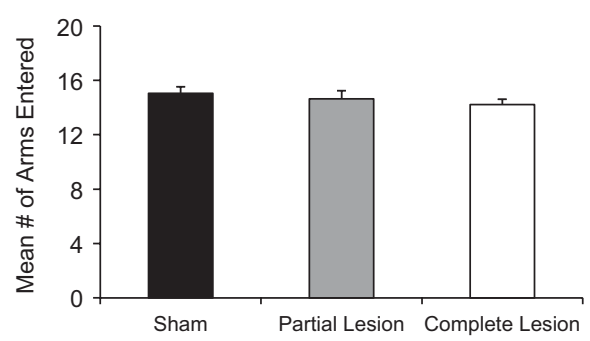

B

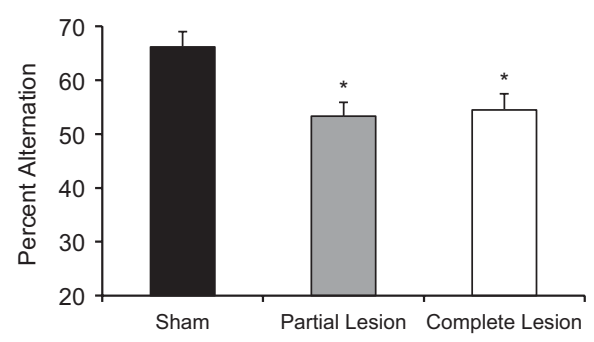

Figure 4. Delayed alternation behavioral results. All rats were tested for two sessions of delayed alternation, but the behavior did note change as a function of session, so the average of the number of arms entered $(A)$ and percent alternation $(B)$ are shown. Neither partial nor complete lesions of the anterior thalamus affected activity level as determined by the mean number of arms entered $(A)$. However, both partial and complete lesions to the anterior thalamus impaired delayed alternation accuracy (mean percent alternation) relative to the sham control group (B). The two lesion groups had a similar degree of impairment as their accuracy scores did not differ from one another. (*) Significant difference from sham group.

was no significant correlation $(r=0.10, P>0.5)$ between delayed alternation performance and ACh efflux. In contrast, there are negative correlations between lesion size and ACh efflux $(r=-0.69, P<0.01)$ (Fig. 6B) and spontaneous alternation performance $(r=-0.66, P<0.01)$ (Fig. 6C) that persisted even when the sham group was removed (both $r^{\prime} \mathrm{s}>-0.54, P<0.05$ ). Although there was a negative correlation between lesion size and delayed alternation when the sham group was considered $(r=-0.53, P<0.01)$ (Fig. 6D), the correlation was not significant $(r=-0.20, P>0.2)$ when only the two ATN lesion groups were considered. The lack of correlations between delayed alternation performance and ACh efflux and lesion extent are because the two lesion groups are equally impaired on that task.

\section{Discussion}

The core diagnostic feature of the amnestic syndrome produced after damage to either the diencephalon (anterior/midline thalamus, mammillary bodies) or medial temporal lobe is often clinically indistinguishable-a severe and persistent loss of new explicit- or episodic-type learning. There are several theories proposed to account for this phenomenon (see Aggleton et al. 2010): (1) the medial temporal lobe and diencephalon have independent, but similar, actions; (2) damage to either region directly down-regulates function in the other region; (3) damage to either the medial temporal lobe or diencephalon causes permanent dysfunction in a third common region that is critical for memory; or (4) a combination of 2 and 3. Our data revealed that damaging the ATN has a dramatic impact on hippocampal function as measured by spatial learning/memory and changes in neurotransmitter levels. Hippocampal-dependent behavior was disrupted to various degrees as a function of ATN lesion extent, but partial or massive ATN damage produced a severe reduction in behaviorally induced activation of ACh. Thus, these data lend credence to the second theory. However, given that our measure of neural activation in the hippocampus (ACh efflux) is not directly controlled by the ATN, another region beyond the hippocampus must also be down-regulated by damage to the ATN. Thus, premise 3 is highly probable.

Acetylcholine is an important excitatory neurotransmitter as ACh levels reflect a general state of activation. In the hippocampus, the majority of ACh release is primarily attributed to the activation of cholinergic neurons located in the MS/DB (Woolf 1991; Gold 2004). The MS/DB modulates hippocampal excitability through the interactions of ACh, GABA, and glutamate (Colom and Garrido-Sanabria 2007). However, the ATN have no direct connections to the MS/DB, but damage to the ATN might reduce the excitatory activation of the hippocampus via other indirect target sites. Indeed, lesions of ATN reduce hippocampal c-Fos immunoreactivity (Jenkins et al. 2002a,b). Similarly, ATN lesions reduce neuronal activation in the retrosplenial cortex (i.e., c-Fos and zif268) (see Jenkins et al. 2004; Poirier and Aggleton 2009), which likely leads to the disruption of hippocampal processing. Similar to the hippocampus, the retrosplenial cortex receives direct cholinergic inputs from the MS/DB and modulates hippocampal activity via dense projections (Gonzalo-Ruiz and Morte 2000). Thus, the altered hippocampal ACh levels after ATN damage could be due to down-regulated neuronal activity in the retrosplenial cortex that interacts with the ATN, MS/DB, and hippocampus.

Our data further confirm that the ATN are critical in a range of hippocampal neural activities. This claim is also supported

\section{A}

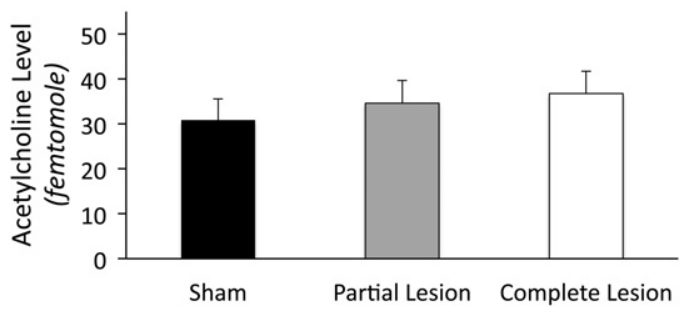

B

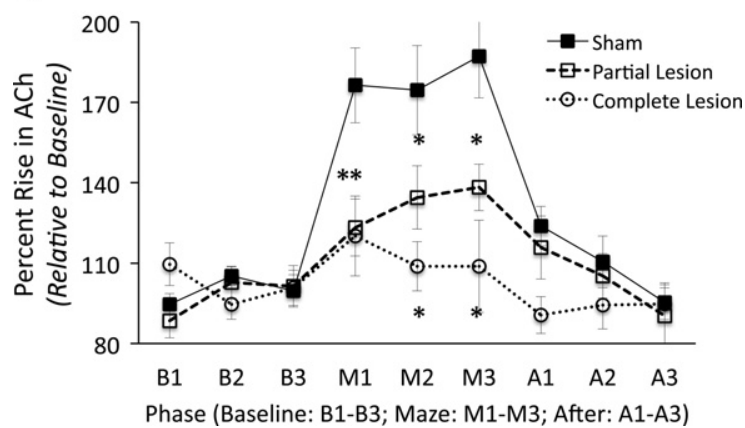

Figure 5. Changes in acetylcholine (ACh) levels. Panel $A$ shows that the groups did not differ in basal levels of ACh in the hippocampus. Panel $B$ reveals the dramatic and significant rise in ACh efflux in the hippocampus from baseline levels (B1-B3) of sham-treated rats during delayed alternation testing $(M 1-M 3)$ and the return to basal levels after testing $(A 1-A 3)$. In contrast, both lesion groups (partial and complete lesions of the anterior thalamus) show a significant blunting of the behaviorally induced increase in ACh efflux. There were no group differences prior to maze testing (B1-B3) or after maze testing (A1-A3). (*) Significant difference from sham group. 
A

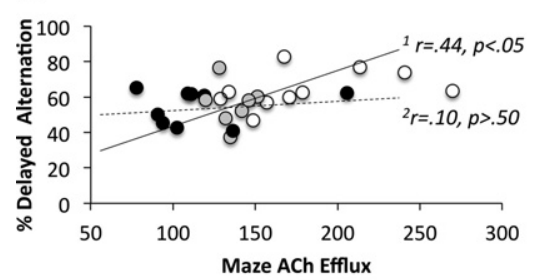

C

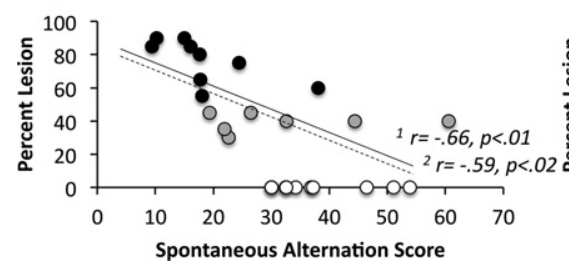

B

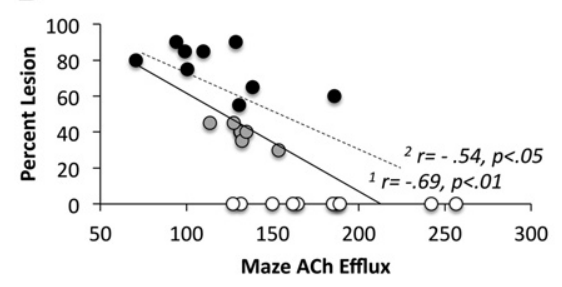

D

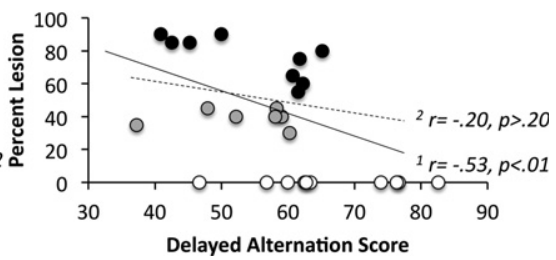

Figure 6. Correlations between behavior, $\mathrm{ACh}$ efflux, and lesion extent. $(A)$ There was a significant positive correlation $(r=0.44, P<0.05)$ between delayed alternation performance and ACh efflux in the hippocampus when all groups were included. However, if the sham group was removed from the analysis, the correlation was not significant. When all three groups were included in the regression, the extent of lesion was negatively correlated with $(B)$ ACh efflux $(r=-0.69, P<0.01),(C)$ spontaneous alternation $(r=-0.66, P<0.01)$, and $(D)$ delayed alternation $(r=-0.53, P<0.01)$. However, if only the two ATN lesion groups were considered in the regression analysis, lesion extent only correlated with ACh efflux and spontaneous alternation performance (both $r>-0.54, P<$ $0.05)$. ( $\left.{ }^{1} r\right)$ All groups are included: sham, partial ATN lesion, and complete ATN; $\left({ }^{2} r\right)$ two ATN lesion groups only, with no sham group. Two trend lines are shown on each graph: The solid lines are the combined regression analyses for the sham (white circles), partial ATN lesion (gray circles), and complete ATN (black circles) groups, whereas the dashed lines are the trend lines when only the two ATN lesion groups are considered (no sham group).

by gene-activated studies (Jenkins et al. 2002b), contralateral disconnection studies (Warburton et al. 2000, 2001), along with electrophysiological evidence. Lesions of the AD disrupt the establishment of head-direction signals in the postsubiculum (Goodridge and Taube 1997) and degrade place-field representations in hippocampal place cells (Calton et al. 2003). In addition, it has recently been demonstrated that the AV nuclei are entrained to hippocampal theta activity (Vertes et al. 2001; Tsanov et al. 2011). Theta-frequency $(4-12 \mathrm{~Hz})$ activity within the hippocampus and associated regions are modulated by the MS region and are correlated with successful spatial navigation and memory functions (Glasgow and Chapman 2007). Such results have led some to conclude that the AV nuclei are part of a MS theta system that modulates neural activation within the extended hippocampal system (Vann and Aggleton 2004; Vertes et al. 2004).

A question is, given that the ATN has no direct interaction with the MS, how would they interact with one another? There are two indirect routes through which this interaction could occur. One proposition is that the medial mammillary bodies may be a critical relay station for theta, transferring information between the MS, thalamus, and hippocampus (Kirk and Mackay 2003; Vann 2010). Consequently, loss of ATN neurons could cause the mammillary bodies to atrophy to some degree (see Tschampa et al. 2011), and such damage could possibly attenuate the rate of communication between the mammillary bodies and the MS. The second is hypoactivity of the retrosplenial cortex after ATN lesions could result in decreased feedback to the MS/DB. It has been found that after the retrosplenial cortex was injected with horseradish peroxidase (HRP), fibers and terminal-like structures positive for HRP were found in the neuropil of the MS/DB, suggesting that the MS/DB neurons may be reciprocally innervated by descending fibers from the retrosplenial cortex (Gonzalo-Ruiz and Morte 2000). Either type of distal dysfunction

could provide an indirect route for neuropathology in the ATN to decrease MS-induced behaviorally stimulated cholinergic release in the hippocampus. However, at this point, we cannot determine the exact mechanism for decreased ACh efflux after ATN lesions.

We found graded effects based on the severity of damage to the ATN on both hippocampal-dependent behavior and ACh efflux. Our classification of complete lesion to the ATN resulted in the broadest behavioral impairment, as both spontaneous alternation and delayed alternation were impaired. Although partial ATN lesions had no effect on spontaneous alternation behavior, they produced comparable deficits to the complete lesion group on the delayed alternation task. There are a few features of these two hippocampal-dependent tasks that may produce differences in sensitivity to neuropathology. First, spontaneous alternation is a nonrewarded task, whereas delayed alternation is a rewarded task. Thus, the motivation levels between the tasks differ. Second, it has been stated that delayed alternation is more susceptible to proactive interference based on the repetition of trials, and proactive interference can be exacerbated by delay intervals (Asin and Fibiger 1984; Bubser and Schmidt 1990). Third, spontaneous alternation can be performed using either egocentric or allocentric information (Futter and Aggleton 2006). The same is true for rewarded alternation; however, after a delay interval the use of egocentric information is disrupted (Futter and Aggleton 2006). Extensive damage to the head-direction cells of the ATN in the complete ATN lesion group would likely result in a greater deficit on a task that could be solved using multiple strategies.

Given the fact that there is no significant difference in activity between the groups, it appears that motivation differences are not likely the major contributor of impairment. Therefore, it may be the case that even partial lesions to the ATN significantly affect task performance when proactive interference and memory load are high.

In contrast, the changes in behaviorally activated ACh efflux were hierarchical relative to lesion site: whereas the sham rats had the greatest behaviorally induced rise over baseline, at an average of $80 \%$, partial ATN lesion rats had an $\sim 35 \%$ rise, whereas complete ATN lesion rats had a blunted behaviorally driven rise in ACh efflux (17\%). Thus, it appears that the greater the destruction of the ATN, the greater the deficit in hippocampal ACh efflux. Interestingly, there was a significant correlation between hippocampal ACh efflux and delayed alternation accuracy.

Although some studies have found that unilateral lesions to the ATN can affect hippocampal function (Jenkins et al. 2002b), other lesion studies have found that the spatial deficit associated with anterior thalamic damage depends on the involvement of all three nuclei (Aggleton et al. 1996; Byatt and Dalrymple-Alford 1996; van Groen et al. 2002). Our data demonstrate that extensive bilateral damage to all three nuclei created the most extensive behavioral and neurochemical impairment, but even partial lesions can degrade hippocampal neural activity and some measures of spatial memory. 
Our past work using the PTD model of Korsakoff Syndrome has revealed that the extended damage to the diencephalon leads to hippocampal dysfunction as revealed by blunted behaviorally evoked ACh efflux (Savage et al. 2003; Roland et al. 2008; Vetreno et al. 2008). In the PTD model only the AVVL nucleus of the ATN complex shows significant neuronal loss, as the AD and ADV are spared (Langlais and Savage 1995; Anzalone et al. 2010). However, there is also critical cell loss in the intralaminar nuclei as well as the medial mammillary nucleus. Furthermore, cholinergic stimulation of the septohippocampal pathway reduces or eliminates the PTD-induced behavioral deficit (Roland et al. 2008, 2010; Roland and Savage 2009). Thus, similar to other neurological diseases, decreased ACh levels induced by thiamine deficiency appear to play a role in the behavioral dysfunction, and increasing ACh levels can lead to a full or partial recovery of cognitive functions.

The site-specific lesioning of the ATN produced a very similar behavioral and neurochemical profile to the PTD model. Our initial hypothesis was that the loss of cholinergic cells in the MS/DB was the critical mediator for the changes in ACh efflux in the PTD model. Indeed, there is a correlation between hippocampal ACh release and cholinergic fiber number in the hippocampus in PTD rats (Anzalone et al. 2010). However, the current lesion data has led us to re-evaluate that conclusion. If discrete lesions to the ATN can dramatically affect hippocampal ACh efflux, then damage to this region-or even the medial mammillary bodies-in the PTD model can also contribute to blunted behaviorally relevant ACh efflux. Mammillary body lesions in mice have been shown to disrupt levels of both basal and behavioral-activated cholinergic markers in the hippocampus (Beracochea et al. 1995).

In summary, although selective damage to the ATN affects hippocampal-dependent behaviors, such damage also substantially decreases behaviorally relevant hippocampal ACh efflux. A critical point from our data and others (see Goodridge and Taube 1997) is that spatial processing is dependent on reciprocal projections from the ATN to the hippocampus. Diaschisis effects can occur when there are anatomical links, both direct and indirect, between structures. However, the distal dysfunction may not be a simple loss of afferent stimulation but rather a complex change in configuration of connectivity and activation (Aggleton et al. 2011). The cholinergic dysfunction data advocate for the position that other brain regions can modulate the input from ATN to the hippocampal formation. The medial mammillary bodies are the primary suspects, as they are one of the few structures that exhibit connectivity between the ATN, the MS, and the hippocampus. Our data support a view that spatial learning and memory is highly dependent on intact circuitry between several critical brain regions (thalamus, hippocampus, mammillary bodies). Specifically, a discrete lesion to ATN has the ability to influence ACh neurotransmission in the hippocampus during maze testing but not at rest. Thus, damage to the ATN did not reduce overall cholinergic tone within the hippocampus; rather the interdependency between the ATN and hippocampus is revealed only during spatial exploration.

\section{Materials and Methods}

\section{Subjects}

Thirty adult male Sprague-Dawley rats (3-5 mo; 300-400 g) served as subjects. Rats were housed one per cage in a colony room with ad libitum access to food and water prior to surgery and were kept on a 12-hr/12-hr light-dark cycle (light cycle onset at 7:00 AM). Every effort was made to minimize animal suffering and the number of animals used. All experiments were conducted according to the National Institutes of Health (NIH) Guide for the Care and Use of Laboratory Animals (NIH Publications No. 8023, revised 1996). All experimental procedures were approved by the Binghamton University Institutional Animal Care and Use Committee (IACUC).

\section{Surgery}

Rats were first randomly assigned to one of the following treatments: ATN lesion $(n=20)$ or sham lesion $(n=10)$. Before surgery, each rat was given an induction dosage of $0.3 \%$ isofluorane vapor (Butler Animal Health Supply) to induce general anesthesia and were then maintained at a $0.2 \%$ dosage for the duration of the surgery. Once deeply anesthetized, it was placed into a stereotaxic instrument (David Kopf Instruments) with a flat skull position and the incisor bar set $3.0 \mathrm{~mm}$ below the interaural line. Bilateral ATN lesions were created by infusing $0.2 \mu \mathrm{L}$ of the neurotoxin N-Methyl-D-asparatate (NMDA, $0.25 \mathrm{M}$ ) from a $5-\mu \mathrm{L}$ Hamilton syringe (Hamilton Company) into sites of reference relative to bregma $[(\mathrm{AP})=-0.17 \mathrm{~mm},-0.21 \mathrm{~mm},(\mathrm{ML})= \pm 0.12 \mathrm{~mm}$, $\pm 0.15 \mathrm{~mm},(\mathrm{DV})=-0.61,-0.56,-0.60)$ (Paxinos and Watson 2004). A similar procedure was used for the sham controls, except there was no chemical infusion. In all rats, a guide cannula (CMA\# 830-9018, CMA Microdialysis Inc.) was placed in the hippocampus [sites: $(\mathrm{AP})=-0.53 \mathrm{~mm},(\mathrm{ML})=-0.51 \mathrm{~mm},(\mathrm{DV})=-0.42 \mathrm{~mm}$ ]. Acrylic cement and three skull screws were used to hold the guide cannula in place. Following surgery, each rat received $0.2 \mathrm{cc}$ of $10 \mathrm{mg} / \mathrm{mL}$ of gentamicin (APP Pharmaceuticals, LLC) as an infection prophylaxis and $0.3 \mathrm{cc}$ of $0.05 \mathrm{mg} / \mathrm{mL}$ buprenorphine (buprenorphine hydrochloride, Hospira Inc.) for analgesia. Immediately after surgery, each rat was placed in a warm incubator until it regained an upright posture. Twenty-four hours later, all rats received a second dose of buprenorphine. One week following surgery, rats were handled daily for 5 min 5 days prior to behavioral testing. Furthermore, 1 wk following recovery, food was restricted to three pellets per rat until it reached $85 \%$ of its free-feeding body weight.

\section{Spontaneous alternation}

On the first day of behavioral testing, each rat was placed on a plus-maze with all sides open allowing full access to arms. The maze had clear Plexiglas sidewalls $(12 \mathrm{~cm}$ high) and the arms of equal distance $(55 \mathrm{~cm})$ that were constructed of wood and painted black. The maze was elevated $80 \mathrm{~cm}$ from the floor, and the testing room contained many extramaze spatial cues. Activity was recorded for a period of $18 \mathrm{~min}$ in regard to arms chosen. An alternation score was determined by recording the number of different arms (minimum $=1$, maximum $=4$ ) entered during a four-choice sequence, dividing the number of different arms entered by four and multiplying that fraction by 100 . Chance performance of this task is $9.3 \%$ [(4/4) $(3 / 4)(2 / 4)(1 / 4)=0.093]$. Upon completion of $18 \mathrm{~min}$ of maze testing, rats were transferred back to their home cage and returned to the colony room.

\section{Delayed alternation and microdialysis}

For delayed alternation, a T-maze was used that had clear Plexiglas sidewalls (12 cm high) with the two goal arms of equal distance $(55 \mathrm{~cm})$ and a start arm with a length of $66 \mathrm{~cm}$. The arms were made of wood painted black, and the maze was elevated $80 \mathrm{~cm}$ from the floor. Transparent Plexiglas guillotine doors that slid into the maze separated the start box (SB) and the two goal boxes (GB) from the choice area.

On each of the two delayed alternation testing sessions, each rat was transported to the testing room and placed in a microdialysis holding cage (acrylic cage-30 $\mathrm{cm} \times 40 \mathrm{~cm}$, depth $35 \mathrm{~cm}$ ) with wood shavings at the bottom). A probe (CMA-3 $\mathrm{mm}$ ) was inserted into the hippocampus. The microdialysis probe was connected to a microinfusion system (CMA/400 pump) and perfused continuously at a rate of $2 \mu \mathrm{L} / \mathrm{min}$ with artificial cerebrospinal fluid (127.6 mM NaCl, $4 \mathrm{mM} \mathrm{Kcl,} 1.3 \mathrm{mM} \mathrm{CaCl}_{2}$ dihyrate, $1.0 \mathrm{mM}$ glucose, $0.9 \mathrm{mM} \mathrm{MgCl}_{2}, 0.9 \mathrm{mM} \mathrm{NaH} \mathrm{PO}_{4}$, and $2 \mathrm{mM} \mathrm{Na}_{2} \mathrm{HPO}_{4}$ brought to a $\mathrm{pH}$ of 7.0 ) that contained neostigmine bromide (500 nM). The neostigmine bromide (Sigma-Aldrich) was added 
to obtain consistent measurable levels of behaviorally relevant changes in ACh efflux (see Chang et al. 2006). There was a 60 -min period of habituation in the maze room holding cage to the dialysis procedure. Afterward, dialysate samples were collected for every $6 \mathrm{~min}$ (sample volume $=12 \mu \mathrm{L}$ ) for a period of 18 min prior to maze testing. This pre-maze phase was used to determine basal levels of ACh in awake rats.

After the 18-min baseline period, the rat was placed in the start box of the maze. On the first trial of each testing day, each rat was allowed a free choice to enter either the left or right arm of the maze, and either response was rewarded with a 45-mg food pellet (BioServ). After consuming the reward ( $\sim 5 \mathrm{sec}$ ), the rat was manually removed from the goal box and placed back into the start box. There was a 30-sec delay interval between the end of the previous trial and the beginning of the next trial. The rat was expected to alternate into the opposite arm of that previously entered. A correct choice was rewarded with the food pellet. An incorrect response (repeat visit to the previous arm) was not rewarded, and the rat was confined to the goal box for a 10 -sec time-out period before being returned to the start box. The number of trials completed during a session was determined by each rat's pace of maze traversal, consumption time, and number of time outs. As stated in the results section, the average number of trials completed in a single session was $\sim 15$ ( \pm 1.9 S.E.M.). At the completion of the maze training, the rat was removed from the maze and placed back into the microdialysis holding cage for an additional period of $18 \mathrm{~min}$ of post-maze dialysate collection. After the 18-min post-maze period, the probe was removed from the rat's head and placed into a standard solution (100 nM concentration of ACh and choline) to assess recovery rate. This delayed alternation protocol was repeated the next day for a total of $2 \mathrm{~d}$ of microdialysis/behavioral testing. Following collection of brain dialysate, samples were frozen for later analysis using highperformance liquid chromatography (HPLC).

\section{High-performance liquid chromatography}

Acetylcholine output was assayed by an Epison HPLC system (BioAnaltical Systems) with an enzyme reactor. The assay system included an ion exchange microbore analytical column (BAS, MR-8904), microbore ACh/choline immobilized enzyme reactor containing acetylcholinesterase and choline oxidase (BAS MF-8903), an auxillary electrode with a radial flow electrochemical thin-layer cell and 13-mm thin layer gasket, a wired enzyme electrode kit (a redox polymer film containing horseradish peroxidase coated on the surface of a 3-mm glassy carbon working electrode), and a low dispersion injected value with a $10 \mathrm{~L}$ polyether-etherketone loop. Standards $(5 \mu \mathrm{L}$ of 20 and $100 \mathrm{nM}$ of $\mathrm{ACh}+\mathrm{Ch}$ ) were injected before and after samples to determine stability of detection. The detection level of ACh was $\sim 10 \mathrm{fmol}$.

\section{Histology}

Following the completion of behavioral testing, rats were deeply anesthetized with SleepAway $(1 \mathrm{mg} / \mathrm{kg}$, i.p.; $26 \%$ sodium pentobarbital in $7.8 \%$ isopropyl alcohol and $20.7 \%$ propylene glycol solution; Fort Dodge Animal Health) and perfused with a $4 \%$ solution of paraformaldehyde (Electron Microscopy Sciences) in $0.1 \mathrm{M}$ phosphate buffer (Sigma-Aldrich). Brains were removed and placed in the $4 \%$ paraformaldehyde solution for $2 \mathrm{~d}$, followed by a $30 \%$ sucrose solution for an additional $3 \mathrm{~d}$. Brains were then frozen and sliced at $40 \mu \mathrm{m}$ with a microtome, beginning from the frontal cortex and ending just prior to the brainstem.

The extent of ATN lesions was determined using immunohistochemistry for the neuronal marker NeuN. Briefly, free-floating sections were agitated in Tris-buffered saline (TBS), and then exogenous peroxidase activity was quenched in $0.3 \%$ hydrogen peroxide. After three TBS washes, slices were blocked and permeabilized using a blocking solution containing $10 \%$ normal horse serum in TBS. Slices were then incubated in primary Neuronal Nuclei (NeuN) antibody (monoclonal mouse anti-NeuN antibody, 1:500; Millapore) for $48 \mathrm{~h}$ and then incubated again in the secondary antibody on the third day (biotinylated horse anti-mouse
IgG, 1:500; Vector Labs). After several TBS washes, the sections were incubated for $2 \mathrm{~h}$ in 1:200 avidin-biotin complex (ABC elite; Vector Laboratories) and visualized using $0.001 \%$ diaminobenzidine in $0.0004 \% \mathrm{H}_{2} \mathrm{O}_{2}$ solution (Sigma-Aldrich). The reaction was stopped by TBS rinses, and sections were mounted and dried on gelatin-coated slides and cover-slipped.

Brain sections that revealed the microdialysis cannula were mounted and stained with Pyronin Y (Electron Microscopy Sciences) for location verification. Only animals with accurate cannula placement within the sectors of the hippocampus were included in the data analysis.

\section{Lesion verification}

Extent of damage was determined on a series of 5-6 serial coronal sections per subject stained with NeuN throughout the anterior and midline thalamus. Percent tissue loss was measured by contour tracing the area absent of NeuN staining on images from a digital rodent atlas (Paxinos and Watson 2004) by an investigator who was blind to the treatment of rats. Corresponding percent lesion size was calculated as the ratio of the total lesion area to the total area of the anterior thalamus. Estimated percent sum of pathology from the AD, AVDM, and AVVL nuclei was used in regression analyses regarding extent of pathology.

\section{Acknowledgments}

This research was funded by grants NINDS 054272 and ARRA NINDS 054272-S1 to L.M.S.

\section{References}

Aggleton JP, Sahgal A. 1993. The contribution of the anterior thalamic nuclei to anterograde amnesia. Neuropsychologia 31: 1001-1019.

Aggleton JP, Hunt PR, Nagle S, Neave N. 1996. The effects of selective lesions within the anterior thalamic nuclei on spatial memory in the rat. Behav Brain Res 8: 189-198.

Aggleton JP, O'Mara SM, Vann SD, Wright NF, Tsanov M, Erichsen JT. 2010. Hippocampal-anterior thalamic pathways for memory: Uncovering a network of direct and indirect actions. Eur I Neurosci 31: 2292-2307.

Aggleton JP, Dumont JR, Warburton EC. 2011. Unraveling the contributions of the diencephalon to recognition memory: A review. Learn Mem 18: 384-400.

Anzalone SJ, Vetreno RP, Ramos RR, Savage LM. 2010. Cortical cholinergic abnormalities during maze exploration following diencephalic damage induced by thiamine deficiency. Eur J Neurosci 32: 847-858.

Asin KE, Fibiger HC. 1984. Spontaneous and delayed spatial alternation following damage to specific neuronal elements within the nucleus medianus raphe. Behav Brain Res 13: 241-250.

Beracochea DJ, Micheau J, Jaffard R. 1995. Alteration of cortical and hippocampal cholinergic activies following lesion of the mammillary bodies in mice. Brain Res 670: 53-58.

Bubser M, Schmidt WJ. 1990. 6-Hydroxydopamine lesion of the rat prefrontal cortex increases locomotor activity, impairs acquisition of delayed alternation tasks, but does not affect uninterrupted tasks in the radial maze. Behav Brain Res 37: 157-168.

Byatt G, Dalrymple-Alford JC. 1996. Both anteromedial and anteroventral thalamic lesions impair radial-maze learning in rats. Behav Neurosci 110: $1335-1348$.

Calton JL, Stackman RW, Goodridge JP, Archey WB, Dudchenko PA, Taube JS. 2003. Hippocampal place cell instability after lesions of the head direction cell network. J Neurosci 23: 9719-9731.

Carlesimo GA, Lombardi MG, Caltagirone C. 2011. Vascular thalamic amnesia: A reappraisal. Neuropsychologia 49: 777-789.

Chang Q, Savage LM, Gold PE. 2006. Microdialysis measures of functional increases in ACh release in the hippocampus with and without inclusion of acetylcholinesterase inhibitors in the perfusate. $J$ Neurochem 97: 697-706.

Colom LV, Garrido-Sanabria E. 2007. Modulation of normal and altered hippocampal excitability states by septal networks. J Neurosci Res $\mathbf{8 5}$ : 2839-2843.

Futter JE, Aggleton JP. 2006. How rats perform spatial working memory tasks: Limitations in the use of egocentric and idiothetic working memory. Quart J Exp Psy 59: 77-99.

Gabriel M, Cuppernell C, Shenker JI, Kubota Y, Henzi V, Swanson D. 1995. Mamillothalamic tract transection blocks anterior thalamic 
training-induced neuronal plasticity and impairs discriminative avoidance behavior in rabbits. J Neurosci 15: 1437-1445.

Glasgow SD, Chapman CA. 2007. Local generation of theta-frequency EEG activity in the parasubiculum. J Neurophysiol 97: 3868-3879.

Gold PE. 2004. Coordination of multiple memory systems. Neurobiol Learn Mem 82: 230-242.

Gold JJ, Squire LR. 2006. The anatomy of amnesia: Neurohistological analysis of three new cases. Learn Mem 13: 699-710.

Gonzalo-Ruiz A, Morte L. 2000. Localization of amino acid, neuropeptised, and cholinergic markers in neuron of the setum-diagonal band complex projecting to the retrosplenial granular cortex of the rat. Brain Res Bull 52: 499-510.

Goodridge JP, Taube JS. 1997. Interaction between the postsubiculum and anterior thalamus in the generation of head direction cell activity. J Neurosci 17: 9315-9330.

Harding A, Halliday G, Caine D, Kril J. 2000. Degeneration of anterior thalamic nuclei differentiates alcoholics with amnesia. Brain 123: $141-154$.

Hasselmo ME. 2006. The role of acetylcholine in learning and memory. Curr Opin Neurobiol 16: 1-6.

Jenkins TA, Dias R, Amin E, Brown MW, Aggleton JP. 2002a. Fos imaging reveals that lesions of the anterior thalamic nuclei produce widespread limbic hypoactivity in rats. J Neurosci 22: 5230-5238.

Jenkins TA, Dias R, Amin E, Aggleton JP. 2002b. Changes in Fos expression in the rat brain after unilateral lesions of the anterior thalamic nuclei. Eur J Neurosci 16: 1425-1432.

Jenkins TA, Vann SD, Amin E, Aggleton JP. 2004. Anterior thalamic lesions stop immediate early gene activation in selective laminae of the retrosplenial cortex: Evidence of covert pathology in rats? Eur J Neurosci 19: 3291-3304.

Kirk IJ, Mackay JC. 2003. The role of theta-range oscillations in synchronizing and integrating activity in distributed mnemonic networks. Cortex 39: 993-1008

Langlais PJ, Savage LM. 1995. Thiamine deficiency in rats produces cognitive and memory deficits on spatial tasks that correlate with tissue loss in diencephalon, cortex, and white matter. Behav Brain Res 68: $75-89$.

Mair RG. 1994. On the role of thalamic pathology in diencephalic amnesia. Rev Neurosci 5: 105-140.

McIntyre CK, Marriott LK, Gold PE. 2003. Patterns of brain acetylcholine release predict individual differences in preferred learning strategies in rats. Neurobiol Learn Mem 79: 177-183.

Mufson EJ, Pandya DN. 1984. Some observations on the course and composition of the cingulum bundle in the rhesus monkey. J Comp Neurol 225: 31-43.

Paxinos G, Watson C. 2004. The rat brain in stereotaxic coordinates. Academic Press, San Diego, CA.

Poirier GL, Aggleton JP. 2009. Post-surgical interval and lesion location within the limbic thalamus determine extent of retrosplenial cortex hypoactivity. Neuroscience 160: 452-469.

Roland JJ, Savage LM. 2007. Hippocampal and striatal acetylcholine efflux during learning in diencephalic-lesioned rats. Neurobiol Learn Mem 87: $123-132$.

Roland JJ, Savage LM. 2009. Blocking GABA-A receptors in the medial septum enhances hippocampal acetylcholine release and maze behavior in a rat model of diencephalic amnesia. Pharm Biochem Behav 92: $480-487$.

Roland JJ, Mark K, Vetreno RP, Savage LM. 2008. Increasing hippocampal acetylcholine levels enhances behavioral performance in an animal model of diencephalic amnesia. Brain Res 1234: 116-127.

Roland JJ, Levinson M, Vetreno RP, Savage LM. 2010. Differential effects of systemic and intraseptal administration of the acetylcholinesterase inhibitor tacrine on the recovery of spatial behavior in an animal model of diencephalic amnesia. Eur J Pharmacol 629: 31-39.
Savage LM, Chang Q, Gold PE. 2003. Diencephalic damage decreases hippocampal acetylcholine release during spontaneous alternation testing. Learn Mem 10: 242-246.

Shibata H. 1993. Direct projections from the anterior thalamic nuclei to the retrohippocampal region in the rat. J Comp Neurol 337: 431445 .

Sutherland RJ, Rodriguez AJ. 1989. The role of the fornix/fimbria and some related subcortical structures in place learning and memory. Behav Brain Res 32: 265-277.

Swanson LW, Cowan WM. 1977. An autoradiographic study of the organization of the efferent connections of the hippocampal formation in the rat. J Comp Neurol 172: 49-84.

Sziklas V, Petrides M. 1999. The effects of lesions to the anterior thalamic nuclei on object-place associations in rats. Eur J Neurosci 11: 559-566.

Tsanov M, Chah E, Vann SD, Reilly RB, Erichsen JT, Aggleton JP, O'Mara SM. 2011. Theta-modulated head direction cells in the rat anterior thalamus. J Neurosci 31: 9489-9502.

Tschampa HJ, Greschus S, Sassen R, Bien CG, Urbach H. 2011. Thalamus lesions in chronic and acute seizure disorders. Neurorad 53: $245-254$

Van der Werf YD, Jolles J, Witter MP, Uylings HB. 2003. Contributions of thalamic nuclei to declarative memory functioning. Cortex 39: 10471062.

Van Groen T, Wyss JM. 1995. Projections from the anterodorsal and anteroventral nucleus of the thalamus to the limbic cortex in the rat. J Comp Neurol 358: 584-604.

van Groen T, Kadish I, Wyss JM. 2002. The role of the laterodorsal nucleus of the thalamus in spatial learning and memory in the rat. Behav Brain Res 136: 329-337.

Vann SD. 2010. Re-evaluating the role of the mammillary bodies in memory. Neuropsychologia 48: 2316-2327.

Vann SD, Aggleton JP. 2004. The mammillary bodies: two memory systems in one? Nat Rev Neurosci 5: 35-44.

Vann SD, Albasser MM. 2009. Hippocampal, retrosplenial, and prefrontal hypoactivity in a model of diencephalic amnesia: Evidence towards an interdependent subcortical-cortical memory network. Hippocampus 19: $1090-1102$.

Vertes RP, Albo Z, Viana Di Prisco G. 2001. Theta-rhythmically firing neurons in the anterior thalamus: Implications for mnemonic functions of Papez's circuit. Neuroscience 104: 619-625.

Vertes RP, Hoover WB, Viana Di Prisco G. 2004. Theta rhythm of the hippocampus: Subcortical control and functional significance. Behav Cogn Neurosci Rev 3: 173-200.

Vetreno RP, Anzalone S, Savage LM. 2008. Impaired, spared, and enhanced ACh efflux across the hippocampus and striatum in diencephalic amnesia is dependent on task demands. Neurobiol Learn Mem 90: 237-244.

Vetreno RP, Klintsova A, Savage LM. 2011. Stage-dependent alterations of progenitor cell proliferation and neurogenesis in an animal model of Wernicke-Korsakoff syndrome. Brain Res 1391: 132-146.

Warburton EC, Baird AL, Morgan A, Muir JL, Aggleton JP. 2000. Disconnecting hippocampal projections to the anterior thalamus produces deficits on tests of spatial memory in rats. Eur J Neurosci 12: $1714-1726$.

Warburton EC, Baird A, Morgan A, Muir JL, Aggleton JP. 2001. The conjoint importance of the hippocampus and anterior thalamic nuclei for allocentric spatial learning: Evidence from a disconnection study in the rat. J Neurosci 21: 7323-7330.

Woolf NJ. 1991. Cholinergic systems in mammalian brain and spinal cord. Prog Neurobiol 37: 475-524.

Received August 10, 2011; accepted in revised form October 12, 2011. 


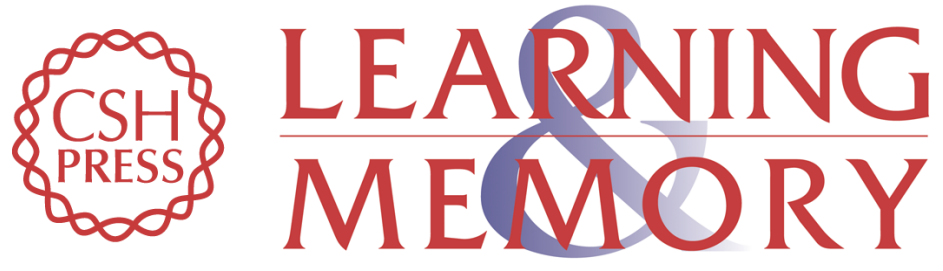

\section{Anterior thalamic lesions alter both hippocampal-dependent behavior and hippocampal acetylcholine release in the rat}

Lisa M. Savage, Joseph M. Hall and Ryan P. Vetreno

Learn. Mem. 2011, 18:

Access the most recent version at doi:10.1101//m.023887.111

References This article cites 55 articles, 9 of which can be accessed free at:

http://learnmem.cshlp.org/content/18/12/751.full.html\#ref-list-1

License

Email Alerting Receive free email alerts when new articles cite this article - sign up in the box at the Service top right corner of the article or click here. 